Cochrane Database of Systematic Reviews

\title{
Pharmacological treatment for continuous spike-wave during slow wave sleep syndrome and Landau-Kleffner Syndrome (Review)
}

Moresco L, Bruschettini M, Calevo MG, Siri L

Moresco L, Bruschettini M, Calevo MG, Siri L.

Pharmacological treatment for continuous spike-wave during slow wave sleep syndrome and Landau-Kleffner Syndrome.

Cochrane Database of Systematic Reviews 2020, Issue 11. Art. No.: CD013132.

DOI: 10.1002/14651858.CD013132.pub2.

www.cochranelibrary.com 
TABLE OF CONTENTS

ABSTRACT 1

PLAIN LANGUAGE SUMMARY

BACKGROUND

OBJECTIVES

METHODS

RESULTS

Figure 1.

DISCUSSION

AUTHORS' CONCLUSIONS

ACKNOWLEDGEMENTS

REFERENCES

CHARACTERISTICS OF STUDIES

APPENDICES

HISTORY

CONTRIBUTIONS OF AUTHORS

DECLARATIONS OF INTEREST

SOURCES OF SUPPORT

DIFFERENCES BETWEEN PROTOCOL AND REVIEW

INDEX TERMS 
[Intervention Review]

\section{Pharmacological treatment for continuous spike-wave during slow wave sleep syndrome and Landau-Kleffner Syndrome}

Luca Moresco ${ }^{1}$, Matteo Bruschettini², Maria Grazia Calevo ${ }^{3}$, Laura Siri4

1Pediatric and Neonatology Unit, Ospedale San Paolo, Savona, Italy. 2Department of Clinical Sciences Lund, Paediatrics, Lund University, Skåne University Hospital, Lund, Sweden. ${ }^{3}$ Epidemiology, Biostatistics Unit, IRCCS, Istituto Giannina Gaslini, Genoa, Italy. ${ }^{4}$ Child Neuropsychiatry Unit, IRCCS Istituto Giannina Gaslini, Genoa, Italy

Contact: Luca Moresco, I.moresco@asl2.liguria.it.

Editorial group: Cochrane Epilepsy Group.

Publication status and date: New, published in Issue 11, 2020.

Citation: Moresco L, Bruschettini M, Calevo MG, Siri L. Pharmacological treatment for continuous spike-wave during slow wave sleep syndrome and Landau-Kleffner Syndrome. Cochrane Database of Systematic Reviews 2020, Issue 11. Art. No.: CD013132. DOI: 10.1002/14651858.CD013132.pub2.

Copyright @ 2020 The Cochrane Collaboration. Published by John Wiley \& Sons, Ltd.

\section{A B S T R A C T}

\section{Background}

Continuous spike-wave during slow wave sleep syndrome (CSWS) and Landau-Kleffner syndrome (LKS) are two epileptic encephalopathies that present with neurocognitive regression, aphasia, and clinical seizures, typically presenting in children around five years of age. The pathophysiology of these conditions is not completely understood. Some studies suggest a common origin for both. No systematic reviews have assessed the efficacy of pharmacological interventions for these conditions.

\section{Objectives}

To assess the benefit and adverse effects of pharmacological interventions for the treatment of CSWS and LKS.

\section{Search methods}

On 8 September 2020, we searched the Cochrane Register of Studies (CRS Web) and MEDLINE Ovid (1946 to September 04, 2020). We applied no language restrictions. CRS Web includes randomised or quasi-randomised, controlled trials from CENTRAL, PubMed, Embase, ClinicalTrials.gov, and the World Health Organization International Clinical Trials Registry Platform.

\section{Selection criteria}

Randomised controlled trials, quasi-randomised controlled trials, and cluster-randomised trials comparing antiepileptic drugs alone, or with steroids or intravenous immunoglobulins, or both versus other antiepileptic drugs, or placebo, or no treatment, administered to children with CSWS and LKS. We planned to compare treatments for the two conditions separately.

\section{Data collection and analysis}

Two review authors independently assessed studies identified by the search strategy for inclusion. The primary outcomes considered in this review were neuropsychological-neurolinguistic functions.

\section{Main results}

Our search strategy yielded 18 references. Two review authors independently assessed all references. We did not find any completed studies to include. We identified one ongoing trial, which was terminated because of lack of enrolment. 


\section{Authors' conclusions}

There was no evidence from trials to support or refute the use of pharmacological treatment for continuous spike-wave during slow wave sleep syndrome or Landau-Kleffner syndrome. Well-designed randomised controlled trials are needed to inform practice.

\section{PLAIN LANGUAGE SUMMARY}

\section{Pharmacological treatment for continuous spike-wave during slow wave sleep syndrome and Landau-Kleffner syndrome}

\section{What was studied in the review?}

Continuous spike-wave during slow wave sleep syndrome (CSWS) is characterised by a regression of mental function and clinical seizures, and usually shows up in children around five years old. Landau-Kleffner syndrome usually shows up in children aged three to eight years, who have previously developed normally. It is characterised by an inability to understand or express language, resulting in decreased communication skills. Treatment may include different drugs (pharmacological treatment), such as antiepileptic drugs and immunotherapies.

\section{What is the aim of this review?}

The aim of this Cochrane Review was to find out the benefit and adverse effects of drug treatment for CSWS and for LKS. To answer this question, we searched scientific databases for randomised controlled trials (clinical studies where people are randomly put into one of two or more treatment groups; these are considered the gold standard for trial design).

\section{Results}

We did not find any relevant, completed studies. We identified one ongoing study, which was terminated because of lack of enrolment.

\section{Conclusions}

There was no evidence to support or refute the use of drug treatment for continuous spike-wave during slow wave sleep syndrome or Landau-Kleffner syndrome.

The evidence is current to September 2020. 


\section{B A C K G R O U N D}

\section{Description of the condition}

Continuous spike and wave during slow wave sleep syndrome (CSWS) is an epileptic encephalopathy that presents with neurocognitive regression and clinical seizures, and demonstrates an electroencephalogram (EEG) pattern of electrical status epilepticus during sleep. It is an age-related condition, typically presenting in children around five years of age, with clinical seizures that progress within two years to a severe epileptic encephalopathy. Electrical status epilepticus during sleep was first described in 1971 as EEG abnormalities that became continuous with sleep onset, and ceased to be continuous upon arousal, occupying at least $85 \%$ of slow sleep tracing (Patry 1971).

Landau-Kleffner syndrome (LKS) is an epileptic encephalopathy that usually manifests itself in children aged three to eight years, with previously normal development (Landau 1957). The main symptoms are acute or subacute aphasia with the inability to recognise, process, or interpret verbal or non-verbal sounds, or both. Nocturnal epileptic activity impedes access to the child's previously established receptive and expressive language (Smith 2003).

These two conditions have many common features, such as: both are age-related conditions; epileptiform discharges localised in particular cortical areas may affect cognitive functions by inhibiting synaptogenesis (Dyhrfjeld-Johnsen 2010; Lengler 2007); cognitive function declines and permanent neuropsychological dysfunction is seen in many cases, although prognosis for seizure control is good; the permanent damage is more evident in patients who had early onset electroencephalographic abnormality, and a prolonged active phase of continuous spike and wave discharges during sleep; if the active phase of paroxysmal activity persists for over two to three years, successful treatment does not resolve neuropsychologic sequelae (Smith 2003). In both conditions, mutations of the same gene, GRIN2A on chromosome 16p13 are found (Lesca 2013). Some study authors consider LKS to be a clinical variant or subtype of encephalopathy related to CSWS, because they both present a common electroencephalographic pattern, characterised by nearly continuous activation of potentially epileptiform discharges during slow-wave sleep, and are known as electrical status epilepticus in slow-wave sleep (ESES) (De Negri 1997; Hughes 2011; Smith 2003; Tassinari 2012). In the last diagnostic scheme of the International League Against Epilepsy, they are considered to be separate entities (Berg 2010).

\section{Description of the intervention}

Since the first description of CSWS, valproic acid (VPA) has been considered the first-line therapy (Patry 1971); recent studies suggest that it might give limited access to critical, period-like plasticity in adult men, as reported by Jervain 2013. Yasuhara 1991 reported on the efficacy of VPA or ethosuximide. Benzodiazepines have been used since the first report of CSWS (Patry 1971). Many studies have been published on the efficacy of clobazam (Larrieu 1986), and diazepam (De Negri 1995; Inutsuka 2006; Kramer 2008).

Some newer antiepileptic drugs, such as levetiracetam, seem to be effective treatments (Aeby 2005; Capovilla 2004; Hoppen 2003; Wang 2008). Other drugs that differ from conventional antiepileptic drugs could also be useful as therapy for CSWS and LKS: trazodone, an antidepressant drug, has been reported in animal models to reduce the amount of cortical plasticity that occurs during slow wave sleep (Aton 2009). Acetazolamide and sulthiame are carbonic anhydrase inhibitors and have been reported to be safe and effective for treatment of CSWS and LKS (Fejerman 2012; Fine 2015).

Many authors have reported the efficacy of steroids and immunoglobulins, especially in cases of failure of conventional antiepileptic drugs (Mikati 2002; Okuyaz 2005; Sinclair 2005).

Choosing an effective drug can be complicated, because of the many adverse effects, such as hepatotoxicity, hypertension, weight gain, and emotional and behavioural side effects.

Other types of interventions have been described for the treatment of CSWS and LKS: vagus nerve stimulation (Carosella 2016), ketogenic diet (Kelley 2016), and neurosurgery (Jeong 2017).

\section{How the intervention might work}

Studies on animals' visual cortices have shown that there is a critical period for cortical development (Crair 2001; Crowley 1999; Crowley 2000). Critical period plasticity can be defined as a window of time in which the cortex develops to a definitive stage, and which might be highly sensitive to different factors. The responses of cortical laminae might change, with a rearrangement of thalamic inputs to cortical layer 4 in the visual cortex (which receives most of the geniculocortical afferents), that propagates through supragranular and infragranular layers (Hubel 1977; Shatz 1978).

However, after the end of the critical period, plasticity is much more limited, with no apparent shift in the thalamocortical inputs (Issa 1999). As cortical areas develop with different time courses, each cortical function has a different critical period. In humans, the critical period for language is important, because it corresponds to the therapeutic window during which treatment might minimise aphasia in LKS (Birdsong 2001; Johnson 1989; Nicholas 2004). Otherwise, CSWS could cause neuropsychological regression, as happens during sleep deprivation in adults (Huber 2004; Urbain 2011).

For all these reasons, it is important to start an effective therapy as soon as possible. The mechanism of action of immunomodulating treatments in childhood epilepsy syndromes is still unknown: some studies report that high levels of inflammatory cytokines, as the activation of the immune system, play a role in ESES syndromes (Lehtimaki 2011; van den Munckhof 2016).

\section{Why it is important to do this review}

The therapy of choice for CSWS and LKS has not been clearly established, and different therapeutic modalities have been tried with conflicting results (Lagae 2009; Van Bogaert 2006). In this Cochrane Review, we aim to assess the evidence regarding the efficacy of pharmacological treatment for CSWS and LKS syndrome.

\section{O B JECTIVES}

To assess the benefit and adverse effects of pharmacological interventions for the treatment of continuous spike and wave during slow wave sleep syndrome (CSWS) and Landau-Kleffner syndrome (LKS). 


\section{METHODS}

\section{Criteria for considering studies for this review}

\section{Types of studies}

We planned to include randomised controlled clinical trials (RCT), quasi-randomised trials, and cluster-randomised trials. We excluded cross-over trials.

\section{Types of participants}

Children, with onset up to 11 years of age, of continuous spike and wave during slow wave sleep syndrome (CSWS) or Landau-Kleffner syndrome (LKS).

\section{Types of interventions}

We included any pharmacological intervention.

We planned to perform comparisons separately for CSWS and LKS.

One or more antiepileptic drugs versus:

- placebo or no intervention;

- other antiepileptic drugs;

- other antiepileptic drugs in association with steroids, immunoglobulin, or both;

- trazodone alone, or in association with other antiepileptic drugs;

- trazodone in association with other antiepileptic drugs, steroids or immunoglobulin, or both;

- acetazolamide alone, or in association with other antiepileptic drugs;

- acetazolamide in association with other antiepileptic drugs, steroids or immunoglobulin, or both;

- ketogenic diet;

- neurosurgery;

- vagus nerve stimulation.

\section{Types of outcome measures}

\section{Primary outcomes}

- Neuropsycological-neurolinguistic function (evaluated by standardised and validated test batteries)

- intelligence quotient (IQ); Wechsler scales (WPPSI III for children between four and six years (Wechsler 2002), WISC IV for children older than 6 years (Wechsler 2005)); Leiter R scale for non-verbal children (Roid 1997)

- attention and memory functions

- executive and visuo-spatial functions (Wisconsin card, Sorting test, Nepsy battery, Tower London test, Rey complex picture test; (Berg 1948)

- language (Peabody picture vocabulary test); (Dunn 2007)

The tests are to be performed at enrolment, after antiepileptic drug therapy, after steroid therapy, before the withdrawal of therapy, or when electroencephalogram (EEG) returns to normal.

\section{Secondary outcomes}

- Improvement of EEG during the sleep (reduction of spike-wave index to under $50 \%$, and return to physiological spindles and kcomplex during sleep), or normalisation

- focal seizures (yes/no)

- focal seizures (reduction from baseline)

- generalised seizures (yes/no)

- generalised seizures (reduction from baseline)

- adverse effects of therapy (e.g. hepatotoxic effects, behavioural modifications, hypertension, weight gain)

- quality of life, measured by validated rating scales (e.g. Modi 2017)

\section{Search methods for identification of studies}

\section{Electronic searches}

We searched the following databases:

- Cochrane Register of Studies (CRS Web; searched 8 September 2020), using the search strategy shown in Appendix 1.

- MEDLINE Ovid (1946 to 4 September 2020), using the search strategy shown in Appendix 2.

CRS Web includes randomised or quasi-randomised, controlled trials from PubMed, Embase, ClinicalTrials.gov, the World Health Organization International Clinical Trials Registry Platform (ICTRP), the Cochrane Central Register of Controlled Trials (CENTRAL), and the Specialized Registers of Cochrane Review Groups, including Epilepsy.

\section{Searching other resources}

We planned to review conference proceedings and systematic reviews, as well as the reference lists from included studies, for other relevant studies.

\section{Data collection and analysis}

Two review authors (LM, LS) independently screened abstracts retrieved through the database search, and planned to extract data and assess for risk of bias. At each stage, we planned to solve disagreements by consensus, or by discussion with a third review author (MGC). We planned to extract data from the published reports, and when necessary, contact the investigators of each trial for missing data.

\section{Selection of studies}

Two review authors (LM, LS) independently screened the titles and abstracts to identify potentially relevant citations, and retrieved the full texts of all potentially relevant articles. We independently assessed study eligibility by completing eligibility forms, designed in accordance with the specified inclusion criteria. We planned to review studies for relevance based on study design, types of participants, interventions, and outcome measures. We planned to resolve any disagreements by discussion, and if necessary, by consulting a third review author (MGC). We planned to list studies excluded after full-text assessment in the 'Characteristics of excluded studies' table with the reasons for exclusion. If the details of the primary trials were unclear, we planned to contact trial authors. 


\section{Data extraction and management}

Two review authors (LM, MGC) planned to independently extract data, using a data extraction form developed for our review and integrated with a modified version of the Cochrane Effective Practice and Organisation of Care Group data collection checklist (EPOC 2013).

We planned to extract the following characteristics from each included study:

- administrative details: author(s); published or unpublished; year of publication; year in which study was conducted; details of other relevant papers cited

- study details: study design; type, duration, and completeness of follow-up (i.e. higher than 80\%); country and location of study, informed consent, and ethics approval

- participant details: sex, age, and number of participants

- intervention details: dose, duration, and class of drug

- outcome details as stated in the 'Types of outcome measures' section

We planned to resolve any disagreement by discussion. We planned to describe ongoing studies identified from our search, where available, detailing the primary author, research question(s), methods, and outcome measures, together with an estimate of the reporting date.

Should queries arise, or in cases where additional data were required, we planned to contact the study investigators or authors for clarification. Two review authors (MGC, MB) planned to use RevMan Web for data entry (RevMan Web 2020).

\section{Assessment of risk of bias in included studies}

Two review authors (MB, MGC) planned to independently assess risk of bias in all the included studies, using the Cochrane tool for assessing risk of bias (Higgins 2011).

We planned to assess the following items:

- selection bias: random sequence generation and selection bias, i.e.

- random sequence generation (biased allocation to interventions) due to inadequate generation of a randomised sequence

- allocation concealment: selection bias (biased allocation to interventions) due to inadequate concealment of allocations prior to assignment

- blinding of participants and personnel: performance bias due to knowledge of the allocated interventions by participants and personnel during the study

- blinding of outcome assessment: detection bias due to knowledge of the allocated interventions by outcome assessors

- incomplete outcome data: attrition bias due to amount, nature, or handling of incomplete outcome data

- selective reporting: reporting bias due to selective outcome reporting

- other bias: bias due to problems not covered elsewhere in the table

We planned to use a 'Risk of bias' graph to illustrate risk across studies. We planned to resolve any disagreements by consensus, and if necessary, by consulting a third review author (LS). See Appendix 3 for a more detailed description of risk of bias for each domain.

\section{Measures of treatment effect}

We planned to extract categorical data for each intervention group and calculate risk ratios (RRs) and absolute risk differences (RDs). We planned to obtain means and standard deviations for continuous data, and perform analyses using mean differences (MDs). For each measure of effect, we planned to also calculate the corresponding $95 \%$ confidence intervals (Cls). We planned to use the standardised mean difference if different scales in test batteries were combined for the primary outcome.

\section{Unit of analysis issues}

The unit of randomisation was the intended unit of analysis (individual child).

If we had found any cluster-RCTs, we would have adjusted them for the designed effect, using the method stated in the Cochrane Handbook for Systematic Reviews of Interventions (Higgins 2011).

\section{Dealing with missing data}

When data were missing, we planned to contact the original study investigators to request the missing data. We planned to obtain a drop-out rate for each study. If we had found a significant drop-out rate (greater than 20\%), we planned to contact the study author(s) for additional data. We planned to perform a sensitivity analysis to evaluate the overall results with and without studies with significant drop-out rates. If a study had reported outcomes only for participants completing the trial, or only for participants who followed the protocol, we planned to contact the study author(s) and ask them to provide additional information, to facilitate an intention-to-treat analysis; and in instances where this was not possible, we planned to perform a complete case analysis.

\section{Assessment of heterogeneity}

We planned to assess clinical heterogeneity by comparing the distribution of important participant factors between trials, and trial factors (randomisation concealment, blinding of outcome assessment, loss to follow-up, treatment type, co-interventions). We assessed statistical heterogeneity by examining the $\mathrm{I}^{2}$ statistic, a quantity that describes the proportion of variation in point estimates that is due to variability across studies, rather than sampling error (Higgins 2011).

We planned to consider the degree of heterogeneity, based on the $\mathrm{I}^{2}$ as follows:

- less than $25 \%$ : no heterogeneity

- $25 \%$ to $49 \%$ : low heterogeneity

- $50 \%$ to $74 \%$ : moderate heterogeneity

- $\geq 75 \%$ : high heterogeneity

We planned to consider statistical heterogeneity to be substantial when $I^{2}$ statistic value was greater than $50 \%$. In addition, we planned to use the $\mathrm{Chi}^{2}$ test of homogeneity, to determine the strength of evidence that the heterogeneity was genuine. If we had detected statistical heterogeneity, we planned to explore possible causes (e.g. differences in study quality, participants, intervention regimens, or outcome assessments) using post-hoc 
subgroup analyses. We planned to consider a threshold of $\mathrm{P}<0.1$ as an indicator of whether heterogeneity (genuine variation in effect sizes) was present.

\section{Assessment of reporting biases}

We planned to investigate publication bias by using funnel plots, if at least 10 clinical trials met the inclusion criteria of the review (Egger 1997; Higgins 2011).

\section{Data synthesis}

We planned to summarise all eligible studies in RevMan Web (RevMan Web 2020). We planned to use standard methodologies for meta-analysis, as described in the Cochrane Handbook for Systematic Reviews of Interventions (Higgins 2011). We planned to calculate the RR and RD, each with $95 \% \mathrm{Cls}$, for categorical outcomes; and the MD with $95 \% \mathrm{Cls}$ for continuous outcomes. For any outcomes for which the included studies were not sufficiently homogeneous, or when insufficient data were available for meta-analysis, we planned to present a narrative synthesis.

If we identify two or more studies for inclusion in future updates, and there is no important clinical or methodological heterogeneity, we will combine them in a meta-analysis. If the studies have no significant statistical heterogeneity, we will use a fixed-effect model for the meta-analysis; however, if there is significant statistical heterogeneity, we will use a random-effects model.

\section{Subgroup analysis and investigation of heterogeneity}

In future updates of this review, If there are sufficient studies, we will stratify comparisons according to:

- different drugs of the same class: for antiepileptic drugs: benzodiazepines (clobazam, diazepam, lorazepam), valproic acid, ethosuximide, sulthiame, levetiracetam. For steroids: ACTH, dexamethasone, methylprednisolone, prednisone, hydrocortisone

- exposure to previous therapies listed in 'Types of interventions' section (yes/no)

- different doses of drugs

\section{Sensitivity analysis}

We planned to conduct sensitivity analyses to explore the effect of the methodological quality of the trials, and to ascertain whether studies at high risk of bias overestimate the effect of treatment.

\section{Summary of findings and assessment of the certainty of the} evidence

We planned to use the GRADE approach to interpret findings (Schünemann 2011). The GRADE approach determines the certainty of the evidence on the basis of an evaluation of five criteria (risk of bias, inconsistency, indirectness, imprecision, publication bias). We planned to use GRADEpro GDT 2020 software, and planned to import data from RevMan Web, to create 'Summary of findings' tables for each comparison included in the review (GRADEpro GDT 2020; RevMan Web 2020). For each comparison, we would have included the following outcomes:

- intelligence quotient (IQ); Wechsler scales (WPPSI III for children between four and six years (Wechsler 2002), WISC IV for children older than 6 years (Wechsler 2005)); Leiter R scale for non-verbal children (Roid 1997)

- attention and memory functions

- executives and visuo-spatial functions (Wisconsin card, Sorting test, Nepsy battery, Tower London test, Rey complex picture test; (Berg 1948))

- focal seizures

- generalised seizures

- adverse effects of therapy (e.g. hepatotoxic effects, behavioural modifications, hypertension, weight gain)

The 'Summary of findings' table for each comparison would have included information on the overall certainty of the evidence from the trials, and information of importance for healthcare decision making.

\section{RE S U L T S}

\section{Description of studies}

\section{Results of the search}

The literature search which we ran in September 2020 identified 18 references (see Figure 1). After screening, we did not consider any trials to be potentially eligible. One trial recruiting children to be randomised to either diazepam or acetazolamide was terminated because of lack of enrolment (NCT02904265; Characteristics of ongoing studies). 
Figure 1.

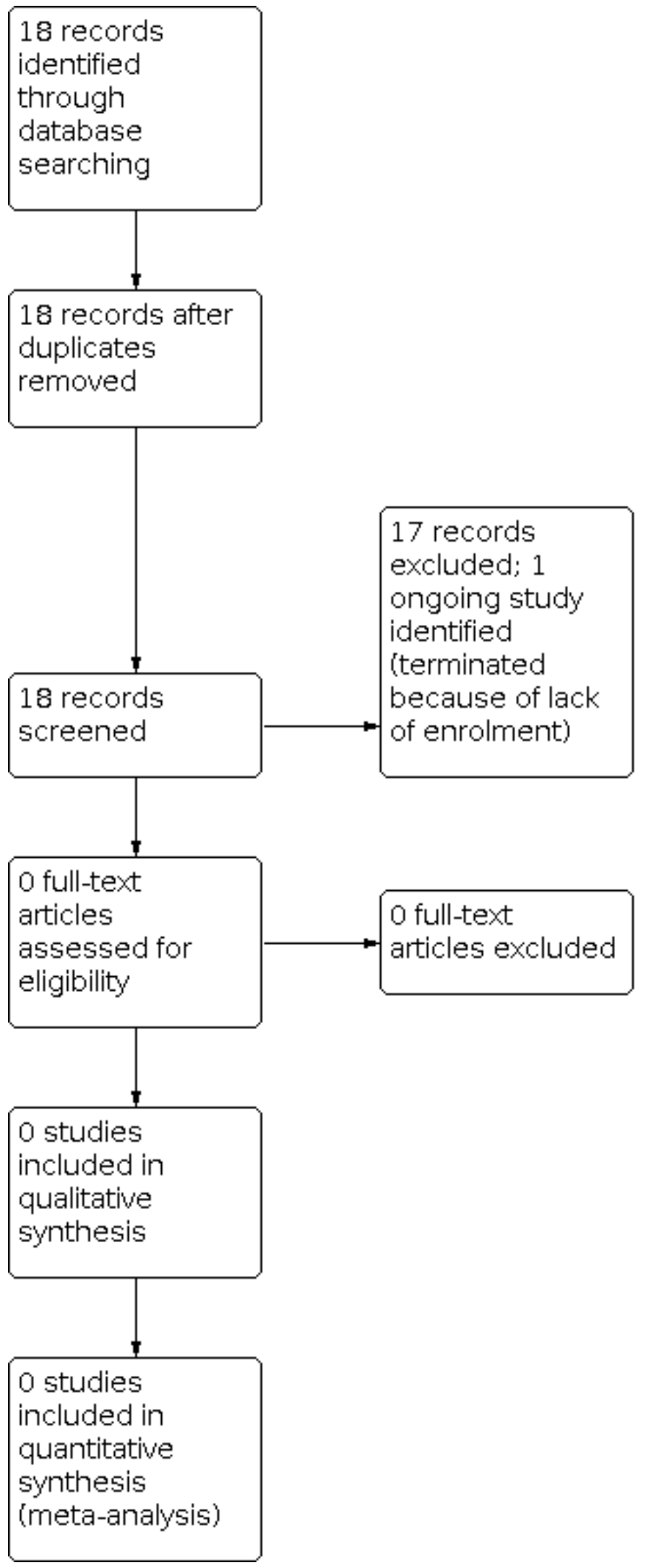




\section{Included studies}

We identified no trials that matched our inclusion criteria.

\section{Excluded studies}

No trials were considered potentially eligible.

\section{Risk of bias in included studies}

We included no studies in this review, hence, no risk of bias is applicable.

\section{Effects of interventions}

No study met the eligibility criteria, hence analyses were not possible. We could not provide a 'Summary of findings' table in the present review.

\section{DISCUSSION}

\section{Summary of main results}

We did not find any randomised controlled trials that assessed the benefit and harms of pharmacological treatment for continuous spike-wave during slow wave sleep syndrome or Landau-Kleffner syndrome in children.

We found one trial recruiting children to be randomised to either diazepam or acetazolamide, which was terminated because of lack of enrolment (NCT02904265).

\section{Overall completeness and applicability of evidence}

We identified no trials for inclusion.

\section{Quality of the evidence}

We identified no trials for inclusion.

\section{Potential biases in the review process}

We used the standard Cochrane methods to conduct this systematic review, therefore, minimising any potential biases. Theoretically, our search strategy was inclusive enough to have included all relevant studies.

\section{Agreements and disagreements with other studies or reviews}

We found no other systematic reviews on the treatment for continuous spike-wave during slow wave sleep and Landau-
Kleffner syndromes. A cross-over study showed that levetiracetam might reduce the spike-wave index on an electroencephalogram during sleep, of children with continuous spike-wave during slow wave and Landau-Kleffner syndromes (Larsson 2012). An open-labelled trial showed that levetiracetam might reduce the frequency of seizures at three and six months in continuous spike-wave during slow wave sleep (Chhun 2011). A retrospective observational study showed a reduction of seizures in continuous spike-wave during slow wave sleep and Landau-Kleffner syndromes during treatment with intravenous methylprednisolone, followed by oral prednisolone, but with high rate of relapse after suspension of treatment (Bakker 2015).

\section{AUTHORS' CONCLUSIONS}

\section{Implications for practice}

There was no evidence from trials to support or refute the use of pharmacological treatment for continuous spike-wave during slow wave sleep and Landau-Kleffner syndromes.

\section{Implications for research}

Randomised controlled trials are needed to evaluate the benefit and harms of pharmacological treatment for continuous spikewave during slow wave sleep and Landau-Kleffner syndromes. Due to the low incidence of the two clinical conditions, multicentre trials are warranted to achieve adequate recruitment of patients. Primary outcomes should include clinically robust outcome measures, such as language development and cognitive impairment.

\section{ACKNOWLEDGEMETS}

This review was supported by the National Institute for Health Research (NIHR), via Cochrane Infrastructure funding to the Epilepsy Group. The views and opinions expressed therein are those of the review authors and do not necessarily reflect those of the Systematic Reviews Programme, the NIHR, the National Health Service (NHS), or the Department of Health and Social Care.

We, and the Cochrane Epilepsy Group are grateful to Sarah Nevitt for providing peer review comments. We also wish to acknowledge the other two peer reviewers who have chosen to remain anonymous. 


\section{R E F E R E N C E S}

\section{References to ongoing studies}

\section{NCT02904265 \{unpublished data only\}}

NCT02904265. Efficacy study of acetazolamide versus diazepam in continuous spike and wave/Landau-Kleffner syndrome [Noninferiority prospective randomized trial of acetazolamide versus diazepam in patients with continuous spike and wave in sleep (CSWS)/Landau Kleffner syndrome (LKS)]. clinicaltrials.gov/ show/NCT02904265 (first received 16 September 2016).

\section{Additional references}

\section{Aeby 2005}

Aeby A, Poznanski N, Verheulpen D, Wetzburger C, Van Bogaert P. Levetiracetam efficacy in epileptic syndromes with continuous spikes and waves during slow sleep: experience in 12 cases. Epilepsia 2005;46(12):1937-42.

\section{Aton 2009}

Aton SJ, Seibt J, Dumoulin MC, Coleman T, Shiraishi M, Frank MG. The sedating antidepressant trazodone impairs sleep-dependent cortical plasticity. PloS One 2009;4(7):e6078. [PMID: 19568418]

\section{Bakker 2015}

Bakker DP, Catsman-Berrevoets CE, Neuteboom RF. Effectiveness of a hybrid corticosteroid treatment regimen on refractory childhood seizures and a review of other corticosteroid treatments. European Journal of Paediatric Neurology: EJPN 2015;19(5):553-60. [PMID: 25976064]

\section{Berg 1948}

Berg EA. Wisconsin card sorting test (WCST) - A simple, objective technique for measuring flexibility in thinking. Journal of General Psychology 1948;39:15-22.

\section{Berg 2010}

Berg AT, Berkovic SF, Brodie MJ, Buchhalter J, Cross JH, van Emde Boas W, et al. Revised terminology and concepts for organization of seizures and epilepsies: report of the ILAE commission on classification and terminology, 2005-2009. Epilepsia 2010;51(4):676-85.

\section{Birdsong 2001}

Birdsong D, Molis M. On the evidence for maturational constraints in second-language acquisition. Journal of Memory and Language 2001;44(2):235-49.

\section{Capovilla 2004}

Capovilla G, Beccaria F, Cagdas S, Montagnini A, Segala R, Paganelli D. Efficacy of levetiracetam in pharmacoresistant continuous spikes and waves during slow sleep. Acta Neurologica Scandinavica 2004;110(3):144-7.

\section{Carosella 2016}

Carosella CM, Greiner HM, Byars AW, Arthur TM, Leach JL, Turner M, et al. Vagus nerve stimulation for electrographic status epilepticus in slow-wave sleep. Pediatric Neurology 2016;60:66-70. [PMID: 27068060]

\section{Chhun 2011}

Chhun S, Troude P, Villeneuve N, Soufflet C, Napuri S, Motte J, et al. A prospective open-labeled trial with levetiracetam in pediatric epilepsy syndromes: continuous spikes and waves during sleep is definitely a target. Seizure 2011;20(4):320-5. [PMID: 21256770]

\section{Crair 2001}

Crair MC, Horton JC, Antonini A, Stryker MP. Emergence of ocular dominance columns in cat visual cortex by 2 weeks of age. Journal of Comparative Neurology 2001;430(2):235-49.

\section{Crowley 1999}

Crowley JC, Katz LC. Development of ocular dominance columns in the absence of retinal input. Nature Neuroscience 1999;2(12):1125-30.

\section{Crowley 2000}

Crowley JC, Katz LC. Early development of ocular dominance columns. Science 2000;290(5495):1321-4.

\section{De Negri 1995}

De Negri M, Baglietto MG, Battaglia FM, Gaggero R, Pessagno A, Recanati L. Treatment of electrical status epilepticus by short diazepam (DZP) cycles after DZP rectal bolus test. Brain \& Development 1995;17(5):330-3.

\section{De Negri 1997}

De Negri M. Electrical status epilepticus during sleep (ESES). Different clinical syndromes: towards a unifying view? Brain \& Development 1997;19(7):447-51.

\section{Dunn 2007}

Dunn LM, Dunn LM. The Peabody Picture Vocabulary Test. 4th edition. Circle Pines, MN: American Guidance Service, 2007.

\section{Dyhrfjeld-Johnsen 2010}

Dyhrfjeld-Johnsen J, Berdichevsky Y, Swiercz W, Sabolek H, Staley KJ. Interictal spikes precede ictal discharges in an organotypic hippocampal slice culture model of epileptogenesis. Journal of Clinical Neurophysiology 2010;27(6):418-24. [PMID: 21076333]

\section{Egger 1997}

Egger M, Davey Smith G, Schneider M, Minder C. Bias in meta-analysis detected by a simple, graphical test. $B M J$ 1997;315(7109):629-34

\section{EPOC 2013}

Cochrane Effective Practice and Organisation of Care (EPOC) Group. Data extraction and management. EPOC Resources for review authors. Oslo: Norwegian Knowledge Centre for the Health Services; 2013. epoc.cochrane.org/epoc-specificresources-review-authors (accessed 15 May 2016).

\section{Fejerman 2012}

Fejerman N, Caraballo R, Cersósimo R, Ferraro SM, Galicchio S, Amartino H. Sulthiame add-on therapy in children with focal epilepsies associated with encephalopathy related to 
electrical status epilepticus during slow sleep (ESES). Epilepsia 2012;53(7):1156-61.

\section{Fine 2015}

Fine AL, Wirrell EC, Wong-Kisiel LC, Nickels KC. Acetazolamide for electrical status epilepticus in slow-wave sleep. Epilepsia 2015;56(9):e134-8.

\section{GRADEpro GDT 2020 [Computer program]}

McMaster University (developed by Evidence Prime) GRADEpro GDT: GRADEpro Guideline Development Tool. McMaster University (developed by Evidence Prime), 2020. Available at gradepro.org.

\section{Higgins 2011}

Higgins JPT, Green S, editor(s). Cochrane Handbook for Systematic Reviews of Interventions Version 5.1.0 (updated March 2011). The Cochrane Collaboration, 2011. Available from training.cochrane.org/handbooks.

\section{Hoppen 2003}

Hoppen T, Sandrieser T, Rister M. Successful treatment of pharmacoresistant continuous spike wave activity during slow sleep with levetiracetam. European Journal of Pediatrics 2003;162(1):59-61.

\section{Hubel 1977}

Hubel DH, Wiesel TN, LeVay S. Plasticity of ocular dominance columns in monkey striate cortex. Philosophical Transactions of the Royal Society B: Biological Sciences 1977;278(961):377-409.

\section{Huber 2004}

Huber R, Ghilardi MF, Massimini M, Tononi G. Local sleep and learning. Nature 2004;430(6995):78-81.

\section{Hughes 2011}

Hughes J. A review of the relationships between LandauKleffner syndrome, electrical status epilepticus during sleep, and continuous spike-waves during sleep. Epilepsy \& Behavior 2011;20(2):247-53.

\section{Inutsuka 2006}

Inutsuka M, Kobayashi K, Oka M, Hattori J, Ohtsuka Y. Treatment of epilepsy with electrical status epilepticus during slow sleep and its related disorders. Brain \& Development 2006;28(5):281-6.

\section{Issa 1999}

Issa NP, Trachtenberg JT, Chapman B, Zahs KR, Stryker MP. The critical period for ocular dominance plasticity in the Ferret's visual cortex. Journal of Neuroscience 1999;19(16):6965-78.

\section{Jeong 2017}

Jeong A, Strahle J, Vellimana AK, Limbrick DD Jr, Smyth MD, Bertrand M. Hemispherotomy in children with electrical status epilepticus of sleep. Journal of Neurosurgery. Pediatrics 2017;19(1):56-62. [PMID: 27791702]

\section{Jervain 2013}

Gervain J, Vines BW, Chen LM, Seo RJ, Hensch TK, Werker JF, et al. Valproate reopens critical period learning of absolute pitch. Frontiers in System Neuroscience 2013;7:102.

\section{Johnson 1989}

Johnson JS, Newport EL. Critical period effects in second language learning: the influence of maturational state on the acquisition of English as a second language. Cognitive Psychology 1989;21(1):60-99.

\section{Kelley 2016}

Kelley SA, Kossoff EH. How effective is the ketogenic diet for electrical status epilepticus of sleep? Epilepsy Research 2016;127:339-43. [PMID: 27710878]

\section{Kramer 2008}

Kramer U, Sagi L, Goldberg-Stern H, Zelnik N, Nissenkorn A, Ben-Zeev B. Clinical spectrum and medical treatment of children with electrical status epilepticus in sleep (ESES). Epilepsia 2008;50(6):1517-24

\section{Lagae 2009}

Lagae L. Rational treatment options with AEDs and ketogenic diet in Landau-Kleffner syndrome: still waiting after all these years. Epilepsia 2009;50(Suppl 7):59-62.

\section{Landau 1957}

Landau WM, Kleffner FR. Syndrome of acquired aphasia with convulsive disorder in children. Neurology 1957;7(8):523-30.

\section{Larrieu 1986}

Larrieu JL, Lagueny A, Ferrer X, Jullien J. Epilepsy with continuous spike-waves during slow sleep: treatment with clobazam [Épilepsie avec décharges continues au cours du sommeil lent: guérison sous clobazam]. Revue d'électroencéphalographie et de neurophysiologie clinique 1986;16(4):383-94. [PMID: 3103177]

\section{Larsson 2012}

Larsson PG, Bakke KA, Bjornaes H, Heminghyt E, Rytter E, Brager-Larsen $L$, et al. The effect of levetiracetam on focal nocturnal epileptiform activity during sleep - a placebocontrolled double-blind cross-over study. Epilepsy \& Behavior 2012;24(1):44-8. [PMID: 22494796]

\section{Lefebvre 2019}

Lefebvre C, Glanville J, Briscoe S, Littlewood A, Marshall C, Metzendorf M-I, et al. Technical Supplement to Chapter 4: Searching for and selecting studies. In: Higgins JPT, Thomas J, Chandler J, Cumpston MS, Li T, Page MJ, et al, editor(s). Cochrane Handbook for Systematic Reviews of Interventions version 6. Cochrane, 2019. Available from www.training.cochrane.org/handbook.

\section{Lehtimaki 2011}

Lehtimaki KA, Liimatainen S, Peltola J, Arvio M. The serum level of interleukin-6 in patients with intellectual disability and refractory epilepsy. Epilepsy Research 2011;95(1-2):184-7.

\section{Lengler 2007}

Lengler U, Kafadar I, Neubauer BA, Krakow K. fMRI correlates of interictal epileptic activity in patients with idiopathic benign focal epilepsy of childhood. A simultaneous EEG-functional MRI study. Epilepsy Research 2007;75(1):29-38. [PMID: 17509833] 


\section{Lesca 2013}

Lesca G, Rudolf G, Bruneau N, Lozovaya N, Labalme A, BoutryKryza N, et al. GRIN2A mutations in acquired epileptic aphasia and related childhood focal epilepsies and encephalopathies with speech and language dysfunction. Nature Genetics 2013;45(9):1061-6.

\section{Mikati 2002}

Mikati MA, Saab R, Fayad MN, Choueiri RN. Efficacy of intravenous immunoglobulin in Landau-Kleffner syndrome. Pediatric Neurology 2002;26(4):298-300.

\section{Modi 2017}

Modi AC, Junger KF, Mara CA, Kellermann T, Barrett L, Wagner J, et al. Validation of the PedsQL Epilepsy Module: a pediatric epilepsy-specific health-related quality of life measure. Epilepsia 2017;58(11):1920-30. [PMID: 28901536]

\section{Nicholas 2004}

Nicholas J, Geers A. Effect of age of cochlear implantation on receptive and expressive spoken language in 3-year-old deaf children. International Congress Series 2004;1273:340-3. [DOI: 10.1016/j.ics.2004.07.043] [Proceedings of the VIII International Cochlear Implant Conference, Indianapolis, IN, 10-13 May 2004]

\section{Okuyaz 2005}

Okuyaz C, Aydin K, Gücüyener K, Serdaroğlu A. Treatment of electrical status epilepticus during slow-wave sleep with highdose corticosteroid. Pediatric Neurology 2005;32(1):64-7.

\section{Patry 1971}

Patry G, Lyagoubi S, Tassinari CA. Subclinical "electrical status epilepticus" induced by sleep in children. A clinical and electroencephalographic study of six cases. Archives of Neurology 1971;24(3):242-52.

\section{RevMan Web 2020 [Computer program]}

The Cochrane Collaboration Review Manager Web (RevMan Web). Version 1.22.0. The Cochrane Collaboration, 2020. Available at revman.cochrane.org.

\section{Roid 1997}

Roid GM, Miller LJ. Leiter International Performance ScaleRevised: Examiners Manual. Stoelting Co; 1997. Stoelting Co, 1997.

\section{Schünemann 2011}

Schünemann HJ, Oxman AD, Vist GE, Higgins JPT, Deeks JJ, Glasziou P, et al. Chapter 12: Interpreting results and drawing conclusions. In: Higgins JP, Green S, editor(s). Cochrane Handbook for Systematic Reviews of Interventions Version 5.1.0 (updated March 2011). The Cochrane Collaboration, 2011. Available from www.training.cochrane.org/handbook..

\section{Shatz 1978}

Shatz CJ, Stryker MP. Ocular dominance in layer IV of the cat's visual cortex and the effects of monocular deprivation. Journal of Physiology 1978;281:267-83.

\section{Sinclair 2005}

Sinclair DB, Snyder TJ. Corticosteroids for the treatment of Landau-Kleffner syndrome and continuous spike-wave discharge during sleep. Pediatric Neurology 2005;32(5):300-6.

\section{Smith 2003}

Smith MC, Hoeppner TJ. Epileptic encephalopathy of late childhood: Landau-Kleffner syndrome and the syndrome of continuous spikes and waves during slow-wave sleep. Journal of Clinical Neurophysiology 2003;20(6):462-72.

\section{Tassinari 2012}

Tassinari CA, Cantalupo G, Dalla Bernardina B, Darra F, Bureau $M$, Cirelli $C$, et al. Chapter 16: Encephalopathy related to status epilepticus during slow sleep (ESES) including Landau-Kleffner syndrome. In: Bureau M, Genton P, Dravet C, Delgado-Escueta A, Tassinari CA, Thomas P, et al, editors(s). Epileptic Syndromes in Infancy, Childhood and Adolescence. 5th edition. Vol. 111. Montrouge, France: John Libbey Eurotext Ltd, 2012:255-75.

\section{Urbain 2011}

Urbain C, Di Vincenzo T, Peigneux P, Van Bogaert P. Is sleeprelated consolidation impaired in focal idiopathic epilepsies of childhood? A pilot study. Epilepsy \& Behavior 2011;22(2):380-4.

\section{Van Bogaert 2006}

Van Bogaert P, Aeby A, de Borchgrave V, De Cocq C, Deprez M, De Tiège $X$, et al. The epileptic syndromes with continuous spikes and waves during slow sleep: definition and management guidelines. Acta Neurologica Belgica 2006;106(2):52-60.

\section{van den Munckhof 2016}

van den Munckhof B, de Vries EE, Braun KPJ, Boss HM, Willemsen MA, van Royen-Kerkhof A, et al. Serum inflammatory mediators correlate with disease activity in electrical status epilepticus in sleep (ESES) syndrome. Epilepsia 2016;57(2):e45-50.

\section{Wang 2008}

Wang SB, Weng WC, Fan PC, Lee WT. Levetiracetam in continuous spike waves during slow-waves sleep syndrome. Pediatric Neurology 2008;39(2):85-90.

\section{Wechsler 2002}

Wechsler D, Cianchetti C. Wechsler Preschool and Primary Scale of Intellegence-III. Giunti O.S. Psycometrics, 2002.

\section{Wechsler 2005}

Wechsler Intelligence Scale for Children- Fourth Edition: Canadian (WISC-IV). Psychological Corporation. ed. Toronto, 2005.

\section{Yasuhara 1991}

Yasuhara A, Yoshida H, Hatanaka T, Sugimoto T, Kobashi Y, Dyken E. Epilepsy with continuous spike-waves during slow sleep and its treatment. Epilepsia 1991;32(1):59-62. 


\section{References to other published versions of this review}

\section{Moresco 2018}

Moresco L, Bruschettini M, Calevo MG, Siri L. Pharmacological treatment for Continuous spike-wave during Slow Wave

\section{CHARACTERISTICS OF STUDIES}

Characteristics of ongoing studies [ordered by study ID]

\section{NCT02904265}

Study name

Non-inferiority prospective randomised trial of acetazolamide versus diazepam in patients with continuous spike and wave in sleep syndrome (CSWS)/Landau-Kleffner syndrome (LKS)

\begin{tabular}{ll}
\hline Methods & Prospective, randomised, parallel, controlled trial \\
\hline Participants & Children: 3 to 12 years old with electrical status epilepticus in sleep and clinical CSWS or LKS \\
Inclusion criteria: & Electrical status epilepticus in sleep and clinical CSWS or LKS, defined by all of the following: spike \\
& wave index $\geq 50 \%$ during first hour of sleep; bilateral synchrony of discharges during sleep; clinical \\
& evidence of behaviour or academic regression, or both; daytime spike wave index $\leq 20 \%$ \\
& Exclusion criteria: \\
- Previous treatment with benzodiazepine or acetazolamide for electrical status epilepticus in & sleep \\
- Current treatment with carbamazepine, phenytoin, oxcarbazepine, phenobarbital, vigabatrin, or & lamotrigine \\
- Antiepileptic medication changes over the month prior to enrolment \\
- Epileptic encephalopathy other than CSWS or LKS \\
- Prior serious adverse reaction to benzodiazepines or acetazolamide \\
- Sulfa allergy \\
- Progressive underlying neurologic condition \\
- Frequent seizures that would prevent the child from maintaining a stable dose of medications \\
- Female participant who has begun menses or is pregnant
\end{tabular}

Interventions

- Diazepam $0.5 \mathrm{mg} / \mathrm{kg}$ (up to maximum $20 \mathrm{mg}$ ) orally, nightly. Duration of therapy is 4 weeks

- Acetazolamide $8 \mathrm{mg} / \mathrm{kg}$ to $10 \mathrm{mg} / \mathrm{kg}$ (up to a maximum dose of $375 \mathrm{mg}$ ) orally, divided twice daily for 1 week, then increased to $11 \mathrm{mg} / \mathrm{kg}$ to $16 \mathrm{mg} / \mathrm{kg}$ (up to a maximum dose of $750 \mathrm{mg}$ ) orally, divided twice daily thereafter. Duration of therapy is 4 to 8 weeks

Outcomes

Primary outcome:

- Spike wave index reduction seen in children on acetazolamide (time frame: within first 4 to 6 weeks after starting therapy with acetazolamide); similar spike wave index reductions (percentage) comparing acetazolamide and diazepam

Secondary outcomes:

- Improvement in behaviours (attention, hyperactivity; time frame: within 4 to 8 weeks of initiation of acetazolamide)

- Improvement in cognitive regression (time frame: within 4 to 8 weeks of initiation of acetazolamide)

- Long-term tolerability to acetazolamide (time frame: 6 months); expect minimal side effects at follow-up at 6 months

Pharmacological treatment for continuous spike-wave during slow wave sleep syndrome and Landau-Kleffner Syndrome (Review) 
NCT02904265 (Continued)

- Short-term tolerability of acetazolamide versus diazepam (time frame: 4 to 8 weeks of start of medications); expect improved side effect profile of acetazolamide compared to diazepam at short-term follow-up

\begin{tabular}{ll}
\hline Starting date & September 2016 \\
\hline Contact information & Katherine C. Nickels, MD, Mayo Clinic Rochester, Minnesota, United States, 55905 \\
\hline Notes & Terminated because of lack of enrolment \\
& clinicaltrials.gov/ct2/show/NCT02904265
\end{tabular}

\section{AP PEN DICES}

\section{Appendix 1. Cochrane Register of Studies (CRS Web) search strategy}

1. (continuous spike ${ }^{\star}$ NEAR2 wave $\left.{ }^{\star}\right)$ :AB,KW,MC,MH,TI AND CENTRAL:TARGET

2. (slow NEAR3 sleep):AB,KW,MC,MH,TI AND CENTRAL:TARGET

3. \#1 AND \#2

4. ("CSWS"):AB,KW,MC,MH,TI AND CENTRAL:TARGET

5. MESH DESCRIPTOR Epilepsy EXPLODE ALL AND CENTRAL:TARGET

6. MESH DESCRIPTOR Seizures EXPLODE ALL AND CENTRAL:TARGET

7. (epilep* OR seizure* OR convuls`):AB,KW,MC,MH,TI AND CENTRAL:TARGET

8. \#5 OR \#6 OR \#7 AND CENTRAL:TARGET

9. \#4 AND \#8

10. MESH DESCRIPTOR Landau-Kleffner Syndrome EXPLODE ALL AND CENTRAL:TARGET

11. (Landau NEAR2 Kleffner):AB,KW,MC,MH,TI AND CENTRAL:TARGET

12. \#10 OR \#11

\#3 OR \#9 OR \#12

\section{Appendix 2. MEDLINE Ovid search strategy}

This strategy includes a modification of the Cochrane Highly Sensitive Search Strategy for identifying randomised trials (Lefebvre 2019).

1. ((continuous spike\$ adj2 wave\$) and (slow adj3 sleep)).tw.

2. "CSWS".tw.

3. exp Epilepsy/

4. exp Seizures/

5. (epilep\$ or seizure\$ or convuls\$).tw.

6.3 or 4 or 5

7. exp *Pre-Eclampsia/ or exp *Eclampsia/

$8.6 \operatorname{not} 7$ 


\section{2 and 8}

10. exp Landau-Kleffner Syndrome/

11. (Landau adj2 Kleffner).tw.

12. 10 or 11

\section{1 or 9 or 12}

14. exp controlled clinical trial/ or (randomi?ed or placebo or randomly).ab.

15. clinical trials as topic.sh.

16. trial.ti.

17. 14 or 15 or 16

18. exp animals/ not humans.sh.

19. 17 not 18

20. 13 and 19

21. remove duplicates from 20

\section{Appendix 3. Assessment of risk of bias in included studies}

Should we identify studies for inclusion in this review in future updates, two review authors (MB, MGC) will independently assess risk of bias in all the included studies using the Cochrane tool for assessing risk of bias (Higgins 2011).

We will assess the following items:

- selection bias: random sequence generation and selection bias, i.e.

- random sequence generation (biased allocation to interventions) due to inadequate generation of a randomised sequence

- allocation concealment: selection bias (biased allocation to interventions) due to inadequate concealment of allocations prior to assignment

- blinding of participants and personnel: performance bias due to knowledge of the allocated interventions by participants and personnel during the study

- blinding of outcome assessment: detection bias due to knowledge of the allocated interventions by outcome assessors

- incomplete outcome data: attrition bias due to amount, nature, or handling of incomplete outcome data

- selective reporting: reporting bias due to selective outcome reporting

- other bias: bias due to problems not covered elsewhere in the table

We will use a 'Risk of bias' graph to illustrate risk across studies. We will resolve any disagreements by consensus, and if necessary, by consulting a third review author (LS).

\section{Random sequence generation (selection bias)}

For each included study, we will categorise the risk of bias regarding random sequence generation as follows:

- low risk: the investigators describe a random component in the sequence generation process, such as referring to a random number table, using a computer random number generator, coin tossing, shuffling cards or envelopes, throwing dice, drawing of lots, minimisation

- high risk: the investigators describe a non-random component in the sequence generation process (sequence generated by odd or even date of birth, sequence generated by some rule based on date or day of admission, sequence generated by some rule based on hospital or clinic record number, allocation by judgment of the clinician, allocation by preference of the participant, allocation based on the results of a laboratory test or a series of tests, allocation by availability of the intervention)

- unclear risk: no or unclear information provided

\section{Allocation concealment (selection bias)}

For each included study, we will categorise the risk of bias regarding allocation concealment as follows: 
- low risk: participant and investigators enrolling participants could not foresee assignment because one of the following, or an equivalent method, was used to conceal allocation: central allocation (including telephone, web-based and pharmacy-controlled randomisation), sequentially numbered drug containers or identical appearance, sequentially numbered sealed opaque envelopes

- high risk: participant and investigators enrolling participants could possibly foresee assignments and thus introduce selection bias, such as allocation based on open random allocation schedule (e.g. a list of random numbers), unsealed or non opaque envelopes, alternation or rotation, date of birth, case record number

- unclear risk: no or unclear information provided

\section{Blinding of study participants and personnel (performance bias)}

For each included study, we will categorise the methods used to blind study participants and personnel from knowledge of which intervention a participant received as follows:

- low risk of bias: no blinding or incomplete blinding, but the review authors judge that the outcome is not likely to be influenced by lack of blinding; blinding of participants and key study personnel ensured, and unlikely that the blinding could have been broken

- high risk of bias: no blinding or incomplete blinding, and the outcome is likely to be influenced by lack of blinding; blinding of key study participants and personnel attempted, but likely that the blinding could have been broken, and the outcome is likely to be influenced by lack of blinding

- unclear risk: no or unclear information provided

\section{Blinding of outcome assessors (detection bias)}

For each included study, we will categorise the methods used to blind outcome assessors from knowledge of which intervention a participant received as follows:

- low risk of bias: no blinding or incomplete blinding, but the review authors judge that the outcome is not likely to be influenced by lack of blinding; blinding of participants and key study personnel ensured, and unlikely that the blinding could have been broken

- high risk of bias: no blinding of outcome assessment, but the review authors judge that the outcome measurement is not likely to be influenced by lack of blinding; blinding of outcome assessment, but likely that the blinding could have been broken, and the outcome measurement is likely to be influenced by lack of blinding

- unclear risk: no or unclear information provided

\section{Incomplete outcome data (attrition bias)}

For each included study and for each outcome, we will describe the completeness of data including attrition and exclusions from the analysis as follows:

- low risk of bias:

○ no missing outcome data

- reasons for missing outcome data unlikely to be related to true outcome (for survival data, censoring unlikely to be introducing bias)

- missing outcome data balanced in numbers across intervention groups, with similar reasons for missing data across groups

- for dichotomous outcome data, the proportion of missing outcomes compared with observed event risk not enough to have a clinically relevant impact on the intervention effect estimate

- for continuous outcome data, plausible effect size (difference in means or standardized difference in means) among missing outcomes not enough to have a clinically relevant impact on observed effect size

- missing data have been imputed using appropriate methods

- high risk of bias

- reason for missing outcome data likely to be related to true outcome, with either imbalance in numbers or reasons for missing data across intervention groups

- for dichotomous outcome data, the proportion of missing outcomes compared with observed event risk enough to induce clinically relevant bias in intervention effect estimate

- for continuous outcome data, plausible effect size (difference in means or standardized difference in means) among missing outcomes enough to induce clinically relevant bias in observed effect size;

- "as-treated" analysis done with substantial departure of the intervention received from that assigned at randomisation

- potentially inappropriate application of simple imputation

- unclear risk: no or unclear information provided

\section{Selective reporting (reporting bias)}

For each included study, we will describe how we investigated the risk of selective outcome reporting bias and what we found. We will attempt to access all the protocols of the included studies through clinical trials registries (e.g. ClinicalTrials.gov (https://clinicaltrials.gov/), 
the International Standard Randomised Controlled Trial Number (ISRCTN) registry (http://www.controlled-trials.com/)) and direct contact with the study authors.

We will assess the methods as follows:

- low risk: the study protocol is available and all of the study's prespecified (primary and secondary) outcomes that are of interest in the review have been reported in the prespecified way; the study protocol is not available but it is clear that the published reports include all expected outcomes, including those that were prespecified (convincing text of this nature may be uncommon)

- high risk: not all of the study's prespecified primary outcomes have been reported; one or more primary outcomes is reported using measurements, analysis methods or subsets of the data (e.g. subscales) that were not prespecified; one or more reported primary outcomes were not prespecified (unless clear justification for their reporting is provided, such as an unexpected adverse effect); one or more outcomes of interest in the review are reported incompletely so that they cannot be entered in a meta-analysis; the study report fails to include results for a key outcome that would be expected to have been reported for such a study

- unclear risk: no or unclear information provided (the study protocol was not available)

\section{Other potential sources of bias (other bias)}

For each included study, we will describe any important concerns we have about other possible sources of bias (e.g. whether there was a potential source of bias related to the specific study design used).

We will assess whether each study is free of other problems that could put it at risk of bias as follows:

- low risk: the study appears to be free of other sources of bias

- high risk: the study is at least one important risk of bias (e.g. the study had a potential source of bias related to the specific study design used or has been claimed to have been fraudulent or had some other problem)

- unclear risk: there may be a risk of bias, but there is either: insufficient information to assess whether an important risk of bias exists or insufficient rationale or evidence that an identified problem will introduce bias

\section{H ISTORY}

Protocol first published: Issue 9, 2018

Review first published: Issue 11, 2020

\section{CONTRIBUTIONSOF AUTHORS}

LM and MGC reviewed the literature and wrote the manuscript.

MB assisted in the review of literature and in writing of the manuscript.

LS reviewed and commented on the manuscript.

\section{DECLARATIONSOF INTEREST}

LM: none known

MB: none known

MGC: none known

LS: none known

\section{SOURCES OF SUPPORT}

\section{Internal sources}

- Pediatric and Neonatology Unit, Ospedale San Paolo, Savona, Italy

LM is employed by this organization

- Institute for Clinical Sciences, Lund University, Lund; Research \& Development, Section for HTA Analysis, Skåne University Hospital, Lund, Sweden

$M B$ is employed by this organization

- Istituto Giannina Gaslini, Genoa, Italy

MGC is employed by this organization 


\section{External sources}

- National Institute for Health Research (NIHR), UK

\section{DIFFERENCES BETWEEN PROTOCOLANDREVIEW}

None.

\section{N DEX TERMS}

\section{Medical Subject Headings (MeSH)}

Landau-Kleffner Syndrome [ ${ }^{\star}$ drug therapy]; Sleep, Slow-Wave [*drug effects]; Syndrome

\section{MeSH check words}

Child, Preschool; Humans 